\title{
A pilot study to investigate effects of haptic feedback training on injection pressure during simulated regional anaesthesia
}

\author{
Williams DJ, ${ }^{1,2}$ Ford SM, ${ }^{1}$ Kyriazis D ${ }^{1}$ \\ ${ }^{1}$ ABMU NHS Trust, Morriston Hospital, Swansea SA6 6NL, UK \\ ${ }^{2}$ Swansea University, Swansea SA2 8PP, UK
}

Swansea University Prifysgol Abertawe

\section{Background}

Nerve injury is a rare but devastating complication of regional anaesthesia, and is associated with excessive injection pressures.[1] Injection pressures $<26 \mathrm{kPa}$ indicate perineural needle placement, and are not associated with nerve injury: injection pressures $>103 \mathrm{kPa}$ are associated with needle-nerve contact and a high risk of nerve injury. [1, 2]. Commercial devices have been designed to measure and display injection pressure on a visual scale, but these devices are not always available and their use may compete with visual attention required for observation of the patient, needle, and ultrasound machine. It is therefore important for clinicians to develop a reliable tactile sense of injection pressure to avoid the administration of high pressures. Tactile awareness of applied pressures has been shown to be poor in both novice and experienced clinical practitioners; and calibration rapidly diminishes over time. [3] A low cost haptic feedback training device would allow clinicians to undertake high frequency periodic 'refresher' training - ideally immediately prior to performing a clinical procedure to maintain tactile awareness and reduce the risk of nerve injury.

Fig. 1

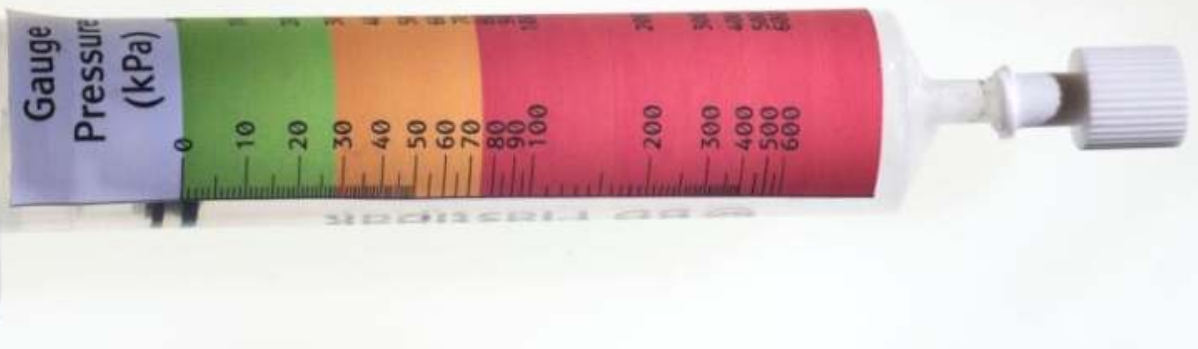

We previously developed a simple low cost device to measure the pressure exerted on a syringe plunger. The device was made from a sealed air-filled $20 \mathrm{~mL}$ syringe calibrated in gauge pressure $(\mathrm{kPa})$ using Boyle's law (Fig. 1), and was validated against an industrial precision pressure transducer. [4] The purpose of this study was to use our device to measure pressures exerted by clinicians during simulated injection of local anaesthetic; and to assess whether a period of training using the device with feedback changed clinicians' behaviour when performing a simulated nerve block.

\section{Method}

Following Research Ethics approval and informed consent, 30 volunteer anaesthetists with a wide range of experience were recruited. Participants were asked to exert what they considered to be a maximum 'safe' injection pressure using our device on five successive attempts. The investigators recorded the maximum pressures, but participants were blinded to the position of the plunger against the scale. Participants were then given the opportunity to practice with the device for as long as they felt necessary, during which time they were able to see the position of the plunger against the scale, indicating whether it would be 'safe' or 'unsafe' in a clinical setting. Following the period of training, participants then performed a further five blinded attempts which were recorded by the investigators.

The mean injection pressure pre- and post-training was found for each participant by taking the truncated mean of each set of five attempts to exclude outliers. Truncated mean injection pressures pre- and posttraining for all participants were plotted as a stacked histogram, and a 2-tailed paired t-test was performed to evaluate the effect of the period of training on the injection pressures exerted by the group.

\section{Results}

300 pressure measurements were collected from 30 participants. A significant reduction in the truncated mean injection pressure exerted by each participant occurred after training (t-statistic: $3.148 ; \mathrm{p}=$ $0.004)$; and the overall mean pressure for the group decreased from $28.0 \mathrm{kPa}$ to $19.6 \mathrm{kPa}$. The number of volunteers exerting mean pressures $>75 \mathrm{kPa}$ fell from one before training to none after training; and the number exerting pressures $>26 \mathrm{kPa}$ fell from eleven before training to three after training. (Fig. 2) .

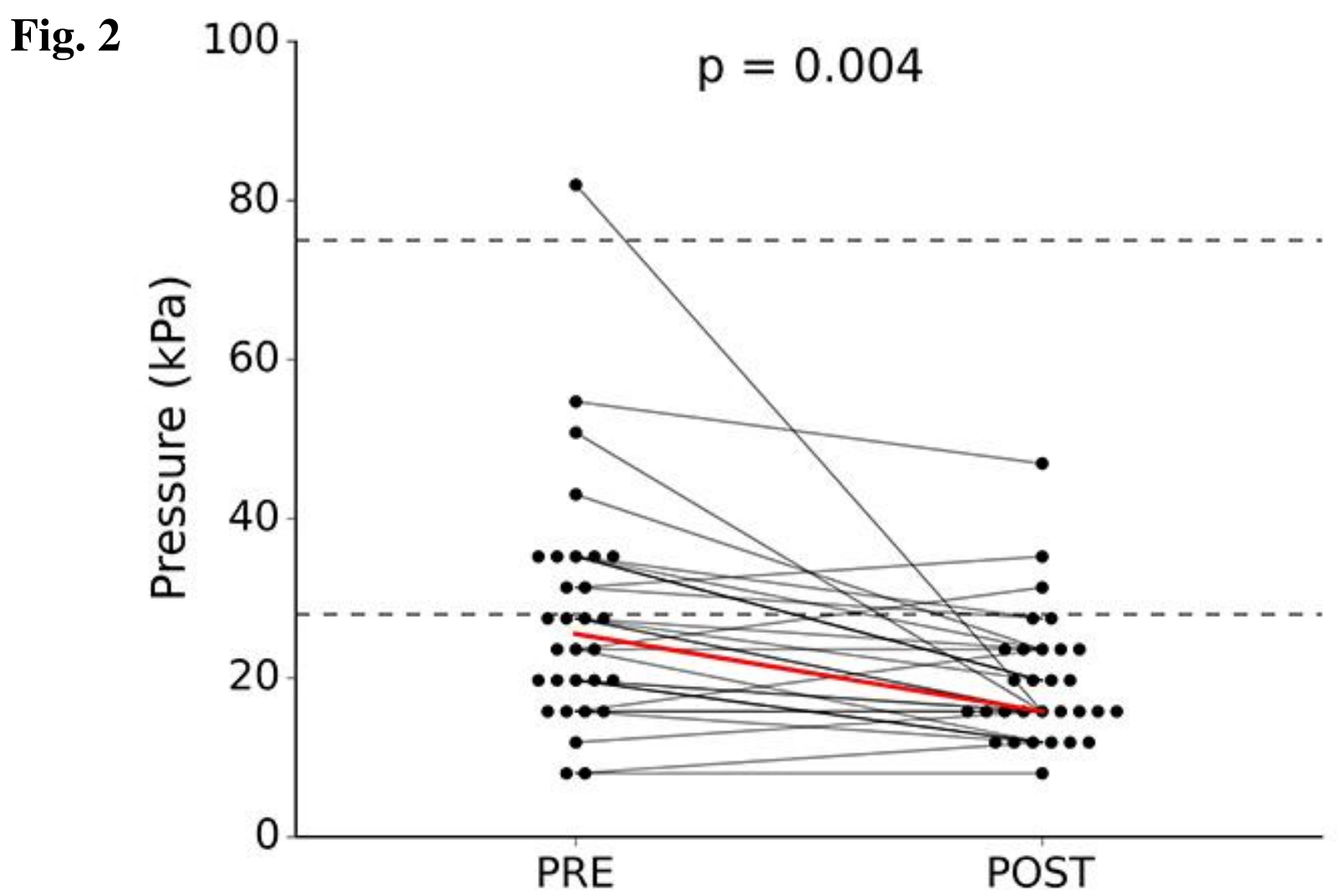

Studies of training for application of cricoid pressure indicate that tactile pressure can be effectively taught using a simple haptic feedback device, but retention of skills is short lived. [3] We would expect a similar rapid degradation of skills with time for regional anaesthesia injection pressures.; however the very low cost and readily availability of our device facilitates near task training and reliable recalibration of subjective assessment of injection pressure immediately prior to performing nerve blocks. Our pilot study did not record the demographics or experience level of the participants. Future studies could explore the effect of these factors on performance, and also investigate the rate of degradation of any performance gains over time (Ebbinghaus's 'forgetting curve').

\section{Conclusions}

$11 / 30$ participants in our study group initially exerted injection pressures which could potentially result in nerve injury. A short period of haptic training with our device immediately prior to simulated injection resulted in improved awareness of and significant reduction in injection pressure. This may transfer to application of lower injection pressures in vivo, and potentially reduce the incidence of nerve injury when performing nerve blocks.

\section{References}

[1] Hadzic A et al. Combination of intraneural injection and high injection pressure leads to fascicular injury and neurologic deficits in dogs.

Reg Anesth Pain Med. 2004; 29(5): 417-23

[2] Gadsden JC et al. Opening Injection Pressure Consistently Detects NeedleNerve Contact during Ultrasound-guided Interscalene Brachial Plexus Block. Anesthesiology. 2014; 120(5): 1246-53

[3] Johnson RL, Cannon EK, Mantilla CB, Cook DA. Cricoid pressure training using simulation: a systematic review and meta-analysis. Br J Anaesth. 2013; 111(3): 338-46

[4] Williams DJ, Ford S. Validation of a low cost training device to limit injection pressure during regional anaesthesia. EJA. 2015; 32(53): 94 